\title{
Models for Interpreting Interstrand \\ Resistance Measurements in Rutherford Cables
}

\author{
M. Neal and V. Kovachev \\ Superconducting Super Collider Laboratory* \\ 2550 Beckleymeade Ave. \\ Dallas, TX 75237 USA
}

March 1994

*Operated by the Universities Research Association, Inc., for the U.S. Department of Energy under Contract No. DE-AC35-89ER40486. 


\subsection{INTRODUCTION}

Variations in the quench behavior of SSC dipole magnets with the current ramp rate have led to studies of the interstrand resistance in real magnet coils. ${ }^{1,2,3}$ These studies involve in-situ measurements of the resistance between pairs of superconducting strands in a keystoned 30 strand Rutherford cable used in SSC dipole magnets. Collared sections slightly longer than one cable twist pitch length are removed from the magnet so that interstrand resistance between one cable twist pitch length of strands can be measured. The magnitude of these measured resistances has been found to be in most cases dependent on the number of strands in line between the input and output strands. The measured values result from current crossing from the input to the output strand along several parallel paths within the multistrand cable. A resistor network model is therefore required for deducing individual contact resistances based on the measured values. The derived contact resistances can then be used to correlate ramp rate quench sensitivity behavior to magnet fabrication and design and as input values for calculating eddy current losses. ${ }^{3}$

In modeling the interstrand resistances in keystoned Rutherford cables the following features should be considered.

- Within one twist pitch length, L, each strand has two crossover contacts with each nonadjacent strand and is in continuous contact with the two adjacent strands.

- Contact between adjacent strands is fundamentally different from contact between crossing strands. Adjacent strands not only are in continuous contact along their lengths but also rollover at the cable edges where the strands are deformed and pressed more tightly together. For these reasons it is expected that interstrand resistance between adjacent strands will typically be less than that between non-adjacent strands. Figure 1 is a sketch of a Rutherford cable showing the difference between crossing and adjacent contact.

- The keystoned shape of the cable probably results in a distribution of resistances at both the crossing and adjacent contacts since compression is greater at the minor edge.

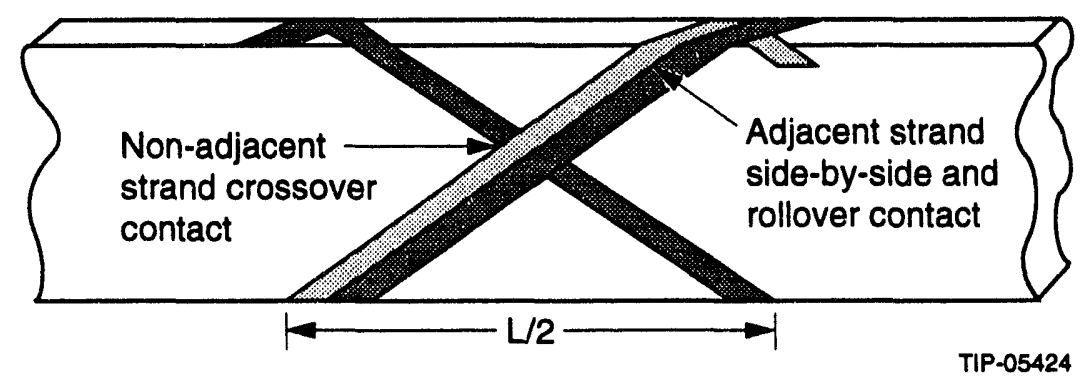

Figure 1. Sketch of a Rutherford cable showing adjacent and non-adjacent interstrand contact.

Three different models have been developed which differ primarily in the degree to which adjacent and nonadjacent contacts are considered separately.

\subsection{MUTUAL EXCLUSION}

In this model the current path between any two strands in the cable is approximated as two parallel resistor networks, one made up of crossover contacts and the other of side-by-side/rollover contacts.

In the first case only crossover contacts are considered. The cable consists of $\mathrm{N}$ strands exactly 1 twist pitch in length, during which each strand pair makes 2 crossover contacts. All contact resistances are 
assumed equal to $r_{c o}$. The two contacts are in parallel so that the interstrand resistance along this direct or primary path is $r_{c_{0}} / 2$. In addition to the primary resistance there are $\mathrm{N}-2$ secondary paths, in which an intermediate strand participates. A secondary resistance is the sum of two primary resistances, or $r_{c o}$. By considering only the primary and all secondary paths all relevant crossover contacts will be considered exactly once. All higher order paths would include at least one contact already considered and so lead to error. There is one primary resistance and N-2 secondary resistances, acting in parallel, so that the total crossover resistance is given by:

$$
\frac{1}{R_{c o}}=\frac{1}{r_{c o} / 2}+(N-2) \frac{1}{r_{c o}}=\frac{N}{r_{c o}}
$$

When only adjacent strands are considered the cable can be modeled as a circular network of resistors in series, where all adjacent resistances are assumed equivalent and equal to $r_{\text {adj }}$. When a voltage is applied across two strands ' $\mathrm{n}$ ' strands apart the current will flow in both the $\mathrm{cw}$ and $\mathrm{ccw}$ directions. The resistance is $n^{*} r_{\text {adj }}$ in the $\mathrm{cw}$ direction, $(\mathrm{N}-\mathrm{n}){ }^{*} \mathrm{r}_{\mathrm{adj}}$ in the $\mathrm{ccw}$ direction. The total interstrand resistance in this case is given by:

$$
\frac{1}{R_{a d j}}=\frac{1}{n r_{a d j}}+\frac{1}{(N-n) r_{a d j}}=\frac{1}{r_{a d j}} \frac{N}{\left(N n-n^{2}\right)}
$$

The measured interstrand resistance is modeled as the result of the crossover and adjacent strand networks in parallel, or

$$
\frac{1}{R_{t o t}}=\frac{1}{R_{c o}}+\frac{1}{R_{a d j}}=\frac{N}{r_{c o}}+\frac{1}{r_{a d j}} \frac{N}{\left(N n-n^{2}\right)}
$$

This is a linear equation in $1 / R_{\text {tot }}$, the measured conductance, and $N /\left(N n-n^{2}\right)$, a strand separation parameter. The individual crossover and adjacent strand contact resistances are then extracted from the $y$ axis intercept and slope of a linear fit to the measured values.

\subsection{LIMITED INCLUSION}

We derive our model by considering first crossover resistance only and then adjacent resistance only and then combining the two networks in parallel. The crossover network consists of the primary resistance (two crossover contacts in parallel)and N-2 secondary resistances, where the secondary resistances are assumed to be two crossover resistances in series. There are four secondary resistances in which one of the two series resistors is actually an adjacent resistor. Making this correction splits the second term of equation 1 into two parts, four parallel resistors, $r_{a}, s e c$, which contain an adjacent resistance:

$$
r_{\mathrm{sec}}^{a}=\frac{r_{c o}}{2}+r_{a d j}
$$

and (N-6) parallel resistors, $\mathrm{r}_{\mathrm{b}, \mathrm{sec}}$, which are unchanged: 


$$
r_{\text {sec }}^{b}=r_{c o}
$$

The primary resistance is still:

$$
r_{p r i}=\frac{r_{c o}}{2}
$$

The total crossover resistance is made up of these three terms in parallel:

$$
\frac{1}{R_{c o}}=\frac{1}{r_{p r i}}+4 \frac{1}{r_{\mathrm{sec}}^{a}}+(N-6) \frac{1}{r_{\mathrm{sec}}^{b}}=\frac{(N-4)}{r_{c o}}+\frac{8}{\left(r_{c o}+2 r_{a d j}\right)}
$$

The total resistance is adjacent and crossover resistance in parallel:

$$
\frac{1}{R_{t o t}}=\frac{(N-4)}{r_{c o}}+\frac{8}{\left(r_{c o}+2 r_{a d j}\right)}+\frac{1}{r_{a d j}} \frac{N}{\left(N n-n^{2}\right)}
$$

This in no way affects the validity of deducing crossover and adjacent resistance from a linear fit, but the value of $r_{c o}$ changes. Ordinarily $r_{a d j}$ is much less than $r_{c o}$ so that $r_{c o}+2 r_{a d j} \approx r_{c o}$ and the first two terms of equation 8 simplify to $(N+4) / r_{c o}$, or $13 \%$ larger than the value of $N / r_{c o}$ given by equation 1 .

\subsection{EXTENSIVE INCLUSION}

The extensive inclusion model uses Kirchoff's Laws to establish a system of interdependant equations to which an iterative solution can be applied to deduce the crossover and adjacent resistances. The contacts considered in this model are all crossover contacts associated with the input strand as well as all adjacent strand contacts. This results in a system of nodes as is shown using a 12 strand cable for illustration in Figure 2. It is assumed that all node output currents are along adjacent strand paths. This assumption is reasonable if crossover resistances are much larger than adjacent strand resistances. Under this condition this model presents a more realistic interplay of adjacent and crossover paths than the previous ones. As before, all adjacent strand resistances are assumed equal to $\mathrm{radj}_{\mathrm{a}}$ and all crossover resistances are assumed $r_{c o}$. There are three paths into the output node so the circuit can be written as three parallel resistors, one a simple crossover resistor and the other two more complex combinations of crossover and adjacent resistors. To determine the crossover and adjacent resistances, a separate equation in $r_{a d j}$ and $r_{c o}$ for several different strand pairs in a given cable turn are set up. The best fit to these equations is achieved by iteration using the measured adjacent strand resistance as the starting point. Results from these calculations generally yielded crossover resistances approximately $30 \%$ smaller than from the mutual exclusion model and adjacent resistances which varied only slightly. 


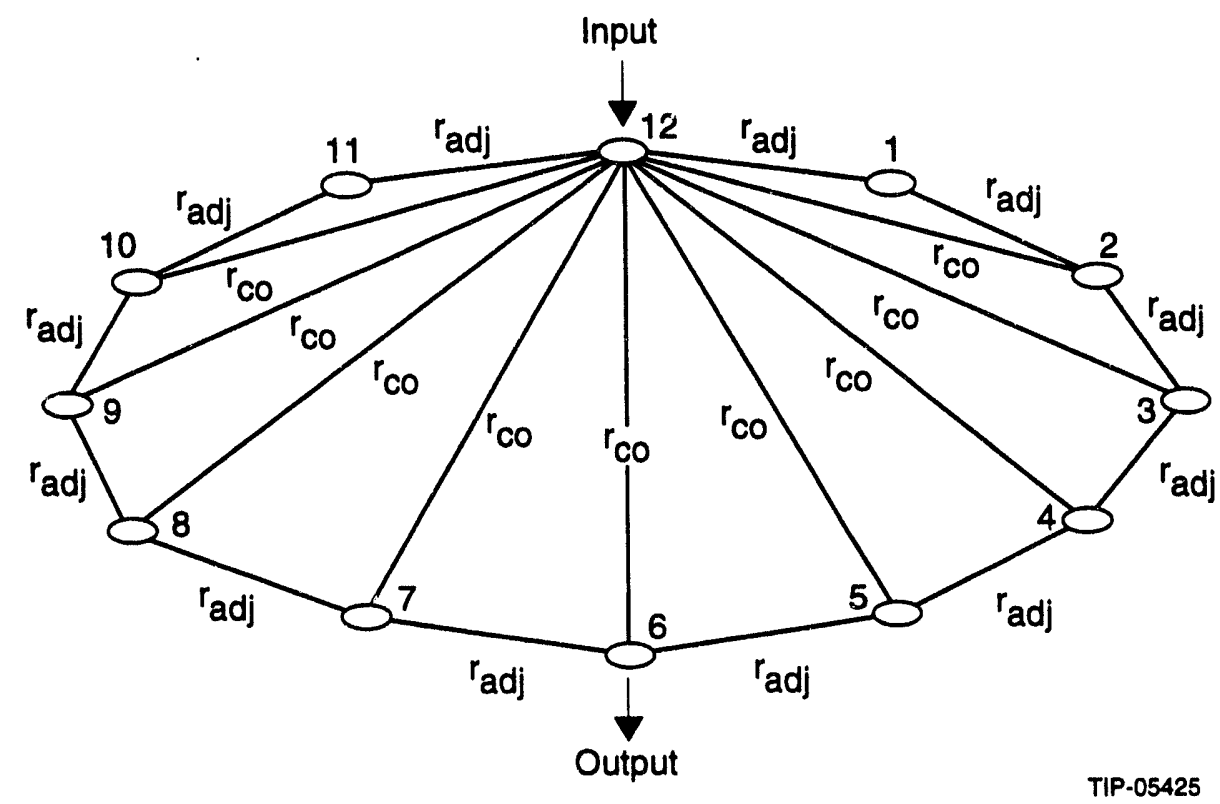

Flgure 2. Schematic of the extensive inclusion circult model for a 12 strand cable. The nodes can be considered to be located within each superconducting strand.

\subsection{CONCLUSIONS}

The mutual exclusion model is a convenient method for determining the approximate value of the average crossover and adjacent strand resistances in a multistrand cable.

The other two methods give corrections of $10-30 \%$, but are less convenient to use and corrections of this magnitude do not provide a qualitatively different evaluation of the cables under test.

All of these methods assume identical adjacent strand resistance and identical crossover resistance. However in reality these resistances can be expected to vary significantly. 


\section{REFERENCES}

1. V.T. Kovachev, M.J. Neal, J.M. Seuntjens, J. Madison, S. Graham, P.G. Cline, M. Wake, and D.W. Capone II, Intrerstrand Resistance of DSA328 Coil, SSCL-637, August 1993.

2. V.T. Kovachev, M.J. Neal, J. Seuntjens, J. Qin, P. Cline, C. Swenson, and D. Capone II, Interstrand Resistance of Selected Sections of DCA312, SSCL-651, November, 1993.

3. V.T. Kovachev, M.J. Neal, D.W. Capone II, J.W. Carr, Jr., and C. Swenson, Interstrand Resistance of SSC Magnets, submitted to Cryogenics, February, 1994. 

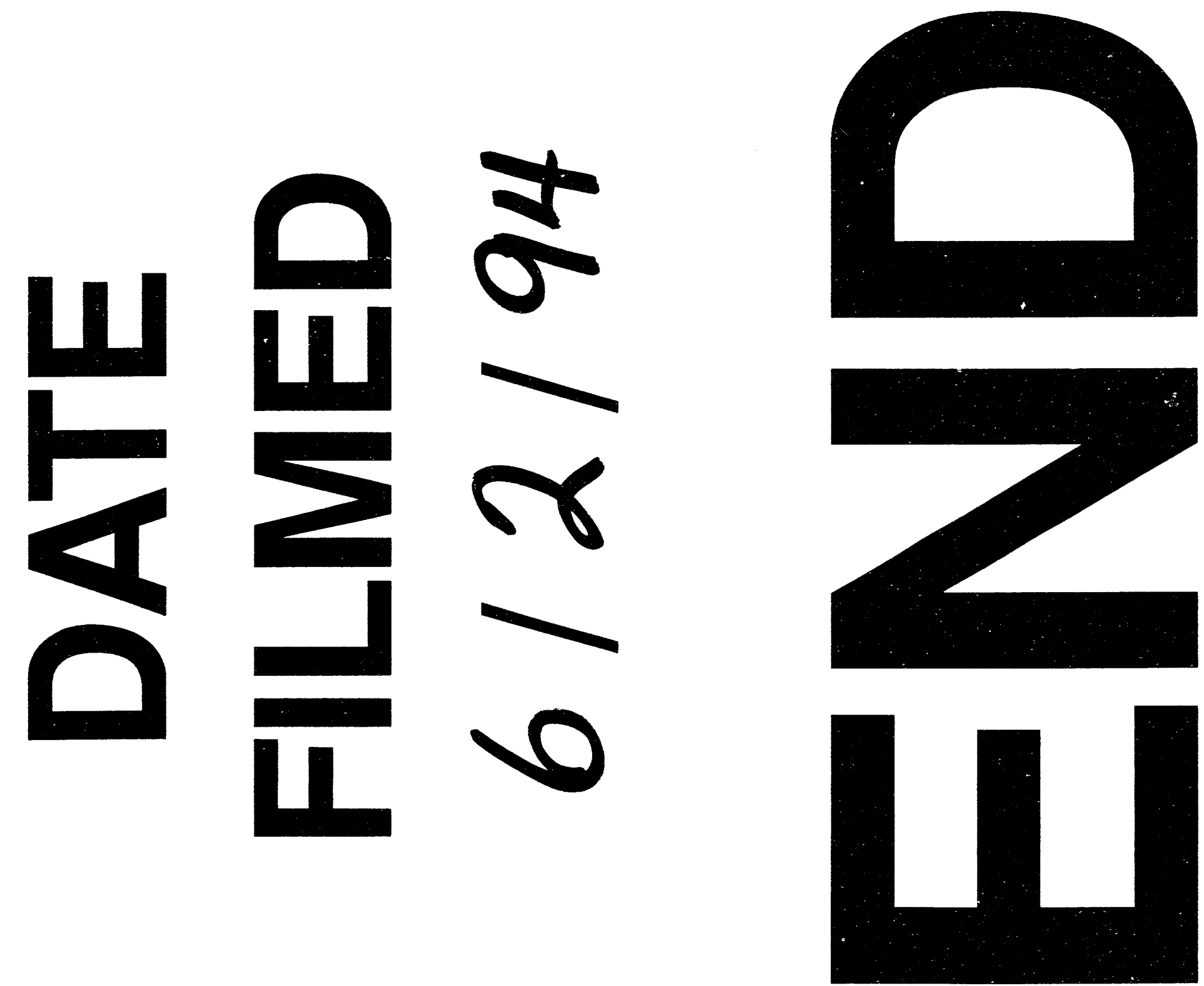
